\title{
Effect of baseline cognitive impairment on association between predicted propofol effect site concentration and Bispectral index or sedation score
}

\author{
Frederick Sieber ${ }^{1 *}$, Karin Neufeld ${ }^{2}$, Esther S. Oh ${ }^{3}$, Allan Gottschalk ${ }^{4}$ and Nae-Yuh Wang ${ }^{5}$
}

\begin{abstract}
Background: This study determined whether the relationship between predicted propofol effect site concentration (Ce) and observer's assessment of alertness/sedation scale (OAA/S) or Bispectral Index (BIS) was similar comparing cognitively intact vs impaired patients undergoing hip fracture repair with spinal anesthesia and sedation.

Methods: Following informed consent baseline mini-mental status exam (MMSE), Clinical Dementia Rating (CDR) and geriatric depression scale (GDS) were obtained. Intraoperatively OAA/S, BIS, and propofol (timing and exact amounts) administered were recorded. Cerebrospinal fluid was collected for Alzheimer's (AD) biomarkers. Mean Ce level (AvgCe) during surgery was calculated using the area under the Ce measurement series from incision to closure, divided by surgical time. Average OAA/S (AvgOAA/S), and BIS (AvgBIS) were similarly calculated. Pearson correlations of AvgCe with AvgOAA/S and AvgBIS were calculated overall and by CDR. Nonparametric locally weighted scatterplot smoothing (LOWESS) fits of AvgOAA/S and AvgBIS on AvgCe were produced, stratified by CDR. Multivariable regression incorporating baseline cognitive measurements or AD biomarkers assessed AvgOAA/S or AvgBIS associations with AvgCe.

Results: In 186 participants AvgBIS and AvgOAA/S correlated with AvgCe (Pearson $\rho=-0.72 ; p<0.0001$ and Pearson $\rho=-0.81 ; p<0.0001$, respectively), and remained unchanged across CDR levels. Association patterns of AvgOAA/S or AvgBIS on AvgCe guided by LOWESS fits and modeled through regression, were similar when stratified by CDR $(p=0.16)$. Multivariable modeling found no independent effect on AvgBIS or AvgOAA/S by MMSE, CDR, GDS, or AD biomarkers after accounting for AvgCe.

Conclusions: When administering sedation in conjunction with spinal anesthesia, cognitive impairment does not affect the relationship between predicted propofol AvgCe and AvgOAA/S or AvgBIS.
\end{abstract}

Keywords: Propofol, Deep sedation, Conscious sedation, Cognitive dysfunction, Hip fractures, Bispectral index, observer's assessment of alertness and sedation (OAA/S), Geriatric anesthesia

\footnotetext{
*Correspondence: fsieber1@jhmi.edu

'Department of Anesthesiology and Critical Care Medicine, Johns Hopkins Bayview Medical Center, 4940 Eastern Avenue, Baltimore, MD 21224, USA

Full list of author information is available at the end of the article
}

(c) The Author(s). 2020 Open Access This article is licensed under a Creative Commons Attribution 4.0 International License, which permits use, sharing, adaptation, distribution and reproduction in any medium or format, as long as you give appropriate credit to the original author(s) and the source, provide a link to the Creative Commons licence, and indicate if changes were made. The images or other third party material in this article are included in the article's Creative Commons licence, unless indicated otherwise in a credit line to the material. If material is not included in the article's Creative Commons licence and your intended use is not permitted by statutory regulation or exceeds the permitted use, you will need to obtain permission directly from the copyright holder. To view a copy of this licence, visit http://creativecommons.org/licenses/by/4.0/ The Creative Commons Public Domain Dedication waiver (http://creativecommons.org/publicdomain/zero/1.0/) applies to the data made available in this article, unless otherwise stated in a credit line to the data. 


\section{Background}

As the population ages, the prevalence of dementia is increasing. Alzheimer's disease (AD), the most common form of dementia in the United States (U.S.), is estimated to affect 5.7 million individuals in 2018, and the annual incidence is expected to double by 2050 [1]. Nearly one third of all people 85 years and older have $\mathrm{AD}$, and it is thought that a large fraction of those that remain undiagnosed possess varying degrees of subclinical $\mathrm{AD}$ pathology [2]. In adults $\geq 65$ years of age undergoing surgery, preoperative cognitive dysfunction, including dementia, is common [3-5]. For instance, in the hip fracture population, dementia prevalence has been reported to be 33\% [6]. Despite the increasing numbers of patients with cognitive impairment undergoing surgery, the literature provides little guidance concerning their anesthetic management. Specifically, it is unclear whether during maintenance of sedation cognitive impairment alters the relationship between predicted effect site concentration and clinically observed Bispectral Index number or sedation score.

Previous investigations examining maintenance anesthetic requirements studied general anesthesia and report no difference in sensitivity to inhalational agents [7] or infusion rate requirements with total intravenous anesthesia [8] comparing patients with and without cognitive dysfunction. While general anesthesia is certainly important to study, the number of procedures utilizing propofol as the primary means of providing procedural sedation or sedation in conjunction with regional anesthesia is enormous. However, there is little information concerning clinical anesthetic dosing requirements in patients with cognitive dysfunction during propofol sedation. We therefore performed a secondary analysis of the STRIDE (A Strategy to Reduce the Incidence of Postoperative Delirium in Elderly Patients) trial data to test the hypothesis that the clinical anesthetic dosing requirements during propofol sedation are similar comparing patients with and without cognitive impairment. The aim of this study was to determine if the relationship between predicted propofol effect site concentration $(\mathrm{Ce})$ and the modified observer's assessment of alertness/sedation scale (OAA/S) [9] or Bispectral index ((BIS) Brain Monitoring System, http://www.medtronic.com/covidien/products/brainmonitoring) number was similar during maintenance of sedation comparing cognitively intact versus cognitively impaired participants.

\section{Methods}

\section{Study design and participants}

The STRIDE trial was a randomized, two-group, parallel, superiority trial whose principal objective was to assess the effectiveness of lighter versus heavier sedation during spinal anesthesia in elderly patients undergoing hip fracture repair. The trial was first registered at ClinicalTrials.gov under registration number NCT00590707 on
1/2008. Johns Hopkins IRB approval was obtained for the prospective STRIDE trial on 9/27/2010 (NA 00041873). All participants provided their written informed consent.

The primary outcome of the STRIDE trial was the impact of the intervention on the incidence of delirium during postoperative Day 1 to Day 5 or to hospital discharge, whichever occurs first. These results have been previously reported $[10,11]$. In short, no overall difference in the incidence of postoperative in-hospital delirium was found between the intervention groups, but a significant effect modification by level of comorbidity was observed, where lighter sedation imparts lower inhospital delirium risk in patients with low pre-operative comorbidity [10]. In addition, when comparing lighter versus heavier sedation, there is no difference in mortality or functional outcomes of elderly hip fracture patients 1 year after surgery [11]. STRIDE was conducted at a single clinical center. A detailed description of the entire trial protocol was published previously in the supplemental material of Li et al. [12].

Briefly, patients $\geq 65$ years old who were undergoing hip fracture repair with spinal anesthesia and propofol sedation and who did not have preoperative delirium or severe dementia were randomized to receive either heavier (OAA/S 0-2) or lighter (OAA/S 3-5) intraoperative sedation. The inclusion criteria were 1) admission to Johns Hopkins Bayview Medical Center for surgical repair of traumatic hip fracture; 2) 65 years of age or older; 3) a preoperative mini-mental status exam (MMSE) [13] score of 15 or higher; and 4) receiving spinal anesthesia. The exclusion criteria included 1) receiving general anesthesia; 2) inability to speak or understand English; 3) severe chronic obstructive pulmonary disease or congestive heart failure; 4) refusal to give informed consent; 5) non-participating attending surgeon; 6) hip fractures in both hips on same admission; 7) repair of another fracture concurrently with the hip fracture; 8) prior hip surgery on the same hip to be repaired in the current surgery; and 9) preoperative delirium.

\section{Data collection at baseline prior to surgery}

Prior to surgery, baseline MMSE, modified Clinical Dementia Rating (CDR) as previously described [14], geriatric depression scale (GDS) [15], and Charlson comorbidity index (CCI) [16] were obtained, in addition to demographic information. CDR was adjudicated by a consensus diagnosis panel [10]. CDR scores were classified as follows: $0=$ normal, $0.5=$ mild cognitive impairment, $\geq 1$ = dementia. Evaluations using Confusion Assessment Method (CAM) [17], Delirium Rating Scale-R-98 (DRS-R98) [18], and abbreviated digit span test (DST) were also collected at baseline and used to confirm absence of preoperative delirium. 


\section{Intervention}

After satisfactory administration of spinal anesthesia, the patient was randomly assigned to one of two groups in blocks with equal allocation, stratified by age and MMSE at baseline. Intra-operatively, one group had the depth of sedation maintained at an OAA/S score of $0-2$. This was the heavier sedation group. Patients in the other group had the depth of sedation maintained at an OAA/ $\mathrm{S}$ score of 3-5. This was the lighter sedation group.

\section{Data collection during surgery}

The propofol was titrated individually for each participant to achieve and maintain the depth of sedation required by that participant's assigned treatment group (lighter or heavier sedation). The depth of sedation for all participants was measured by the OAA/S, administered every 15 min intra-operatively. During the intraoperative study period, the BIS was also recorded. The BIS monitor readout was covered throughout the surgery so that the Study Anesthesiologist/Anesthetist remained masked to the BIS values while administering propofol. The BIS readings served as an independent measure of the level of adherence to the trial interventions. Mean arterial blood pressure (MAP) was measured via oscillotonometry every $5 \mathrm{~min}$, and automatically recorded in the electronic medical record. After surgery, the MAP values were abstracted and entered into the database. After surgery, the BIS values were abstracted, matched in time to their corresponding MAP values, and entered into the database at 5 min intervals. During surgery propofol was administered intravenously using the Alaris PC 8100 series infusion pump which gives continuous output of total volume $(\mathrm{ml})$ infused. A continuous download of total propofol volume infused was obtained for each study case. From this data, the predicted propofol Ce was calculated on a minute by minute basis using the method of Schnider et al. [19]. Ce values were then matched in time to their corresponding MAP values and entered into the database at 5-min intervals.

Mean predicted Ce level (AvgCe) during surgery for each participant was calculated based on the area under the Ce measurement series from incision to end of surgery, divided by the surgery time --- the length of time between incision and end of surgery. Average OAA/S (AvgOAA/S), BIS (AvgBIS) and MAP (AvgMAP) levels during surgery were calculated using the same approach. The Ce values matched in time to the corresponding MAP values were used in the calculation of avgCe. Similarly, the BIS and OAA/S values matched in time to the corresponding MAP values were used in the calculation of avgBIS and avgOAA/S, respectively.

Prior to the administration of spinal anesthesia, approximately $6 \mathrm{cc}$ of cerebrospinal fluid (CSF) was collected and stored for later analysis of AD biomarkers [14].

\section{Statistical analysis}

Distributions of baseline characteristics before surgery and measurements during surgery were described. Mean and standard deviations (SDs) were calculated for continuous variables and frequency distributions (n and \%) were reported for categorical variables. Potential level of systematic bias in using AvgOAA/S and AvgBIS as proxy measures of AvgCe during surgery were assessed using BlandAltman (B-A) plots. Given that AvgOAA/S and AvgBIS were both significantly correlated with AvgCe with negative correlations, reversed variables (5-AvgOAA/S and 100AvgBIS, respectively) were used in the B-A analysis. Due to the differences in range of score for these 3 variables, all three variables were also standardized to have mean $=0$ and $\mathrm{SD}=1$ to produce the B-A plots. Pearson correlations were calculated overall and by CDR and CCI levels. To explore whether the associations between AvgOAA/S or AvgBIS and AvgCe were influenced by cognitive measurement or comorbidity, nonparametric locally weighted scatterplot smoothing (LOWESS) fits of AvgOAA/S and AvgBIS on AvgCe were produced, stratified by the CDR score levels ( 0 , 0.5 , and $\geq 1$ ). Similar LOWESS fits were also derived according to the CCI levels $(0,1,2$, and $>2)$. For ease of interpretation, nonlinear associations suggested by the LOWESS fit were approximated by linear or segmental linear models, as appropriate, in subsequent regression analyses. To better understand the relationship between AvgOAA/S or AvgBIS and AvgCe, multivariable regression analyses incorporating baseline cognitive measurements or CSF biomarkers as potential effect modifiers were performed to assess potential differentiation of associations of AvgOAA/S and AvgBIS with AvgCe by levels of these variables. Analyses were carried out using SAS 9.4, and an estimated association or interaction with $p$-value $<0.05$ was considered statistically significant.

\section{Results}

Table 1 shows the baseline characteristics for this patient group. Exact timing of propofol change in infusion rates and boluses was recorded in 186/200 participants. Thus, we focused the analyses on these 186 participants. BIS data was not recorded in $n=3$ and baseline CDR was not completed in $n=2$ patients. As an ancillary study to the main STRIDE trial, cerebrospinal fluid biomarkers were obtained in $n=178$ patients. No statistically significant difference in baseline characteristics between these 186 participants and those 14 patients who did not have predicted Ce measurement was noted (Table 1).

Systematic bias of OAA/S and BIS measurements against $\mathrm{Ce}$ Over the observed range of AvgOAA/S or AvgBIS, no systematic bias in using them as proxy measures of AvgCe during surgery was found (Fig. 1). The mean 
Table 1 Patient characteristics in Mean (SD) or n (\%) at baseline and measurements during surgery

\begin{tabular}{|c|c|c|}
\hline Baseline characteristics & $\begin{array}{l}\text { With Ce } \\
(n=186)\end{array}$ & $\begin{array}{l}\text { Without Ce } \\
(n=14)\end{array}$ \\
\hline \multicolumn{3}{|l|}{ Before surgery } \\
\hline Age & $81.9(7.8)$ & $79.8(7.0)$ \\
\hline Mini-Mental Status Examination (MMSE) & $24.2(3.7)$ & $25.1(3.4)$ \\
\hline Geriatric depression scale score & $3.9(3.6)$ & $3.0(2.0)$ \\
\hline \multicolumn{3}{|l|}{ Clinical Dementia Rating $(n=184 / 14)$} \\
\hline 0 & $75(41 \%)$ & $7(50 \%)$ \\
\hline 0.5 & $88(48 \%)$ & $6(43 \%)$ \\
\hline$\geq 1.0$ & $21(11 \%)$ & $1(7 \%)$ \\
\hline Charlson Comorbidity Score & $1.5(1.7)$ & $2.0(2.4)$ \\
\hline 0 & $66(35 \%)$ & $6(43 \%)$ \\
\hline 1 & $83(45 \%)$ & $3(21 \%)$ \\
\hline 2 & $26(14 \%)$ & $3(21 \%)$ \\
\hline$>2$ & $11(6 \%)$ & $2(14 \%)$ \\
\hline \multicolumn{3}{|l|}{ Alzheimer's cerebrospinal fluid biomarkers } \\
\hline Total Tau, pg/ml $(n=164 / 6)$ & $\begin{array}{l}490.8 \\
(275.1)\end{array}$ & $\begin{array}{l}617.7 \\
(435.7)\end{array}$ \\
\hline Phosphorylated Tau (pg/ml $(n=163 / 6)$ & $56.7(25.1)$ & $63.0(32.9)$ \\
\hline Amyloid Beta 42 (pg/ml $(\mathrm{n}=164 / 6)$ & $\begin{array}{l}298.3 \\
(164.1)\end{array}$ & $\begin{array}{l}281.2 \\
(106.7)\end{array}$ \\
\hline Amyloid Beta 42/Total Tau(n= 164/6) & $0.71(0.38)$ & $0.61(0.32)$ \\
\hline $\begin{array}{l}\text { Amyloid Beta 42/Phosphorylated Tau } \\
(n=163 / 6)\end{array}$ & $5.75(2.83)$ & $5.16(2.29)$ \\
\hline \multicolumn{3}{|l|}{ During surgery } \\
\hline $\begin{array}{l}\text { Observers Assessment Alertness/Sedation } \\
\text { score }\end{array}$ & $2.2(2.1)$ & $1.4(2.1)$ \\
\hline Bispectral Index number $(n=183 / 13)$ & $69.1(18.1)$ & $75.0(13.7)$ \\
\hline Brain Effect Site Concentration, ug/ml & $1.26(0.79)$ & $---(---)$ \\
\hline Mean Arterial Pressure & 73.4 (10.5) & 77.1 (11.8) \\
\hline
\end{tabular}

CDR, Clinical Dementia Rating $(0=$ normal, $0.5=$ mild cognitive impairment, $\geq 1$ = dementia); MMSE, Mini-Mental Status Examination (maximum score of 30 points)

difference of AvgOAA/S and AvgBIS from AvgCe were both 0 , with $95.2 \%$ of the observed differences within the range of $+/-1.96 \mathrm{SD}$.

\section{BIS determinants}

AvgBIS was strongly correlated with AvgCe during surgery (Pearson $\rho=-0.72 ; p<0.0001$ ), and the correlation remained strong across CDR levels (Table 2).

The correlation seemed the strongest in patients with $\mathrm{CDR}=0$, with a trend toward weaker correlation as cognitive decline measured by CDR worsened. The strong correlation between AvgBIS and AvgCe was also maintained throughout the range of preoperative Charlson co-morbidity scores observed in the STRIDE population (Table 3).
There was no statistically significant differentiation on the level of association between AvgBIS and AvgCe by CDR or CCI levels.

Linear regression modeling demonstrated that AvgBIS was strongly predicted by $\operatorname{AvgCe}(p<.0001)$ and less so by avgMAP $(p=0.01)$. AvgBIS and AvgMAP correlated at $\mathrm{CCI}=0(\mathrm{r}=0.37 ; p=0.002)$. However, at higher $\mathrm{CCI}$ scores correlations were statistically not significant between AvgBIS and AvgMAP (Table 4).

Modest correlations existed between AvgBIS and AvgMAP at mild cognitive impairment $(\mathrm{MCI}, \mathrm{CDR}=0.5$ ) level only.

\section{OAA/S determinants}

AvgOAA/S was strongly correlated with AvgCe during surgery (Pearson $\rho=-0.81 ; p<0.0001$ ), and the correlation remained strong across CDR levels (Table 2). The correlation seemed the strongest in patients with $\mathrm{CDR}=$ 0 , with a trend toward slightly weaker correlation as cognitive decline measured by CDR worsen. The overall correlation between AvgOAA/S and AvgCe was strong and maintained throughout the range of preoperative Charlson co-morbidity scores observed in the STRIDE population (Table 3). AvgOAA/S and AvgMAP did not correlate throughout the range of preoperative $\mathrm{CCI}$ and CDR scores observed in the STRIDE population. Results from linear regression modeling also demonstrates that AvgOAA/S level is strongly predicted by AvgCe $(p<.0001)$, but not by AvgMAP.

\section{Effect of baseline cognition or comorbidity}

Regression analyses based on association patterns suggested by nonparametric LOWESS fits of AvgOAA/S on AvgCe showed no statistically significant difference between association patterns when stratified by either the CDR score levels $(0,0.5$, and $\geq 1 ; p=0.16)$ or the CCI score levels $(0,1,2$, and $\geq 3 ; p=0.37$ ) (Fig. 2, A and C). Similarly, association patterns suggested by LOWESS fits of AvgBIS on AvgCe were not statistically different when stratified by either the CDR score levels $(0,0.5$, and $\geq 1)$ or the CCI score levels $(0,1,2$, and $\geq 3$ ) (Fig. 2, B and D). AvgOAA/S and avgBIS were highly correlated. At CDR $0,0.5$, and 1 or 2 , the Pearson correlation was 0.81 $(p<0.0001), 0.76(\mathrm{p}<0.0001)$, and $0.63(p=0.002)$, respectively. Multivariable modeling found no independent effect on AvgBIS by either MMSE, CDR, CDR sum of boxes, GDS, or any cerebrospinal fluid AD biomarker (data not shown). Other variables tested without independent effect on AvgBIS included age, BMI, and CCI. Regression modeling found no association between AvgOAA/S and either MMSE, CDR, CDR sum of boxes, or any cerebrospinal fluid AD biomarker. Two interactions independently predicted AvgOAA/S. AvgOAA/S was predicted by an age-AvgCe interaction as well as an 
A

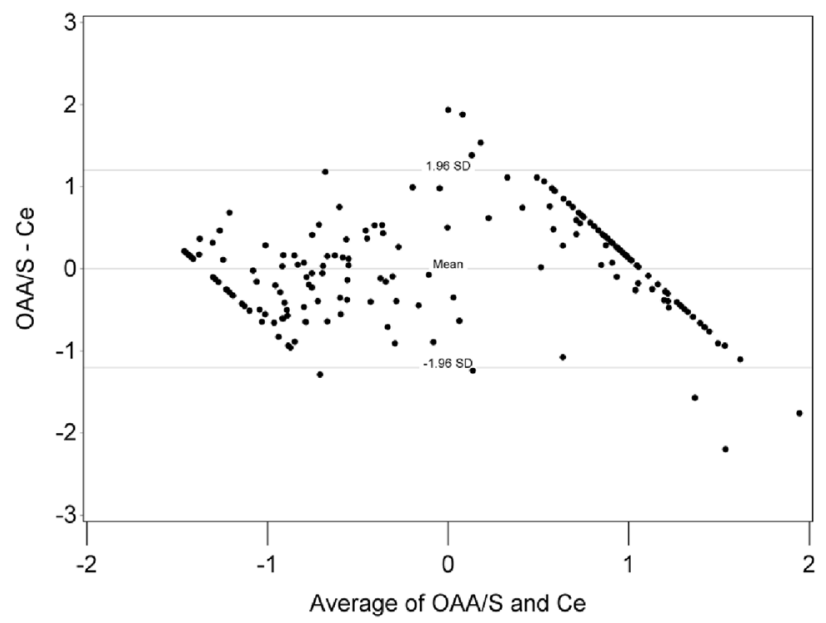

B

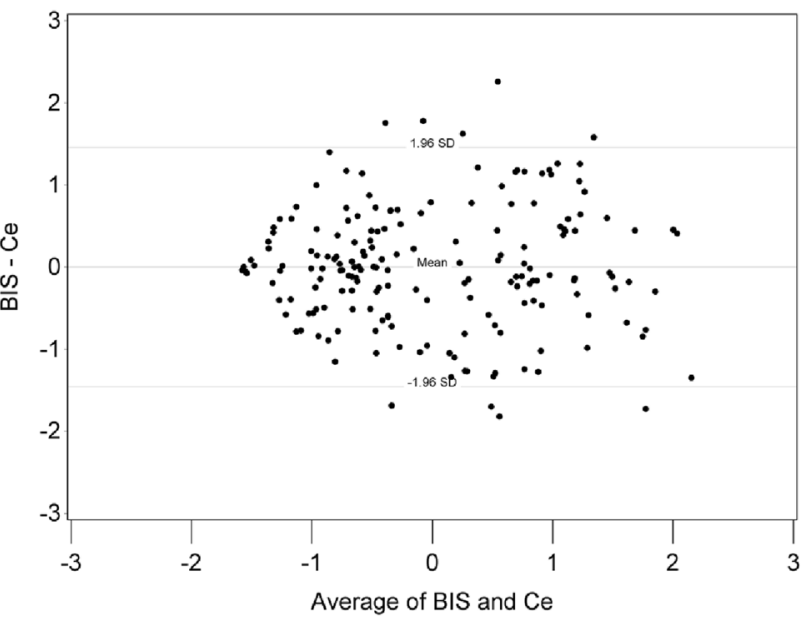

Fig. 1 Bland-Altman plots. a shows Bland-Altman plot for OAA/S and Ce. Y-axis is calculated difference between OAA/S and Ce; X-axis is mean of OAA/S and Ce. OAA/S values were reversed in order (i.e., 5 - OAA/S was used). OAA/S and Ce values were then standardized to have respective distributions both with mean of 0 and standard deviation of 1. b shows Bland-Altman plot for BIS number and Ce. Y-axis is calculated difference between BIS number and Ce; X-axis is mean of BIS number and Ce. BIS values were reversed in order (i.e., 100 - BIS was used). BIS and Ce values were then standardized to have respective distributions both with mean of 0 and standard deviation of 1

age-GDS interaction (Table 5), where the same level of increase in AvgCe was observed with greater decrease in AvgOAA/S score in older patients than in younger patients, and that patients with more severe depressive symptoms before surgery were observed with lower AvgOAA/S score during surgery than patients with less severe depressive symptoms with greater magnitude in this sedation score differences among older patients than in younger patients. Other variables tested without independent effect on AvgOAA/S included BMI, and CCI.

\section{Discussion}

This study did not find evidence to support that the associations between predicted propofol AvgCe and both AvgOAA/S and AvgBIS during sedation among elderly patients undergoing hip fracture repair were significantly altered by baseline CDR or any of the other baseline cognitive variables analyzed.

The current study reports that during sedation, the predicted propofol AvgCe and to a lesser extent

Table 2 Correlations between AvgCe and AvgBIS or Avg OAA/S, overall and by clinical dementia rating

\begin{tabular}{cccccc}
\hline & \multicolumn{1}{c}{ Avg BIS } & & & Avg OAA/S & \\
\cline { 2 - 3 } & Pearson & $\mathrm{P}$ & & Pearson & $\mathrm{P}$ \\
\hline Overall & -0.72 & $<.0001$ & & -0.81 & $<.0001$ \\
\multicolumn{2}{c}{ Clinical Dementia Rating } & & & \\
0 & -0.81 & $<.0001$ & & -0.86 & $<.0001$ \\
0.5 & -0.67 & $<.0001$ & & -0.80 & $<.0001$ \\
$\geq 1$ & -0.58 & 0.0070 & & -0.78 & $<.0001$ \\
\hline
\end{tabular}

AvgMAP affect AvgBIS. The literature reports that BIS, blood propofol concentration [20], Ce [21], and blood pressure are closely related [22]. However, in clinical circumstances where blood pressure decreases occur independent of anesthetic cardiovascular effects, decreases in brain perfusion may be associated with lower BIS numbers independent of anesthetic depth [23-25]. Even less dramatic changes in blood pressure have been associated with BIS modifications. For instance, during stable anesthetic maintenance, blood pressure and BIS are independently correlated $(\mathrm{r}=0.696)$ during positional change from supine to beach chair in preparation for shoulder surgery [26]. The mean BIS level during sedation was not independently affected by any of the baseline cognitive measurements including CDR and CDR sum of boxes, MMSE, or CSF AD biomarker levels. Previous investigations examining the effects of cognitive dysfunction on maintenance anesthetic dosing requirements studied only general anesthesia, used multiple agents and analyzed either retrospective data [7] or

Table 3 Correlations between AvgCe and AvgBIS or Avg OAA/S by level of Charlson co-morbidity scores

\begin{tabular}{|c|c|c|c|c|}
\hline & \multicolumn{2}{|l|}{ Avg BIS } & \multicolumn{2}{|c|}{ Avg OAA/S } \\
\hline & Pearson & $P$ & Pearson & $P$ \\
\hline \multicolumn{5}{|c|}{ Charlson Co-Morbidity Index } \\
\hline 0 & -0.80 & $<.0001$ & -0.86 & $<.0001$ \\
\hline 1 & -0.68 & $<.0001$ & -0.81 & $<.0001$ \\
\hline 2 & -0.76 & $<.0001$ & -0.73 & $<.0001$ \\
\hline$>2$ & -0.61 & 0.05 & -0.94 & $<.0001$ \\
\hline
\end{tabular}


Table 4 Correlations between AvgBIS and AvgMAP, overall and by Charlson score and clinical dementia rating

\begin{tabular}{lll}
\hline & Pearson & $P$ \\
\hline Overall & 0.21 & 0.004 \\
Charlson Co-Morbidity Index & & \\
0 & 0.37 & 0.002 \\
1 & 0.12 & 0.29 \\
2 & 0.14 & 0.50 \\
$>2$ & 0.27 & 0.42 \\
Clinical Dementia Rating & & \\
0 & 0.18 & 0.12 \\
0.5 & 0.31 & 0.004 \\
$\geq 1$ & -0.11 & 0.66 \\
\hline
\end{tabular}

lacked adequate power [8]. In addition, the severity of dementia was incompletely characterized using either baseline MMSE [8] or history of dementia to establish diagnosis [7]. These studies reported no difference in sensitivity to inhalational agents (as defined by BIS only) [7] or infusion rate requirements using the total intravenous combination of remifentanil and propofol [8] during maintenance of general anesthesia when comparing patients by presence or absence of cognitive dysfunction. Our study is unique in its focus on sedation, using more than simply BIS to assess sedation depth, and careful classification of preoperative cognitive state. In addition, the use of a single anesthetic agent is pertinent to procedural sedation which in actual practice is often accomplished with only the single agent propofol.

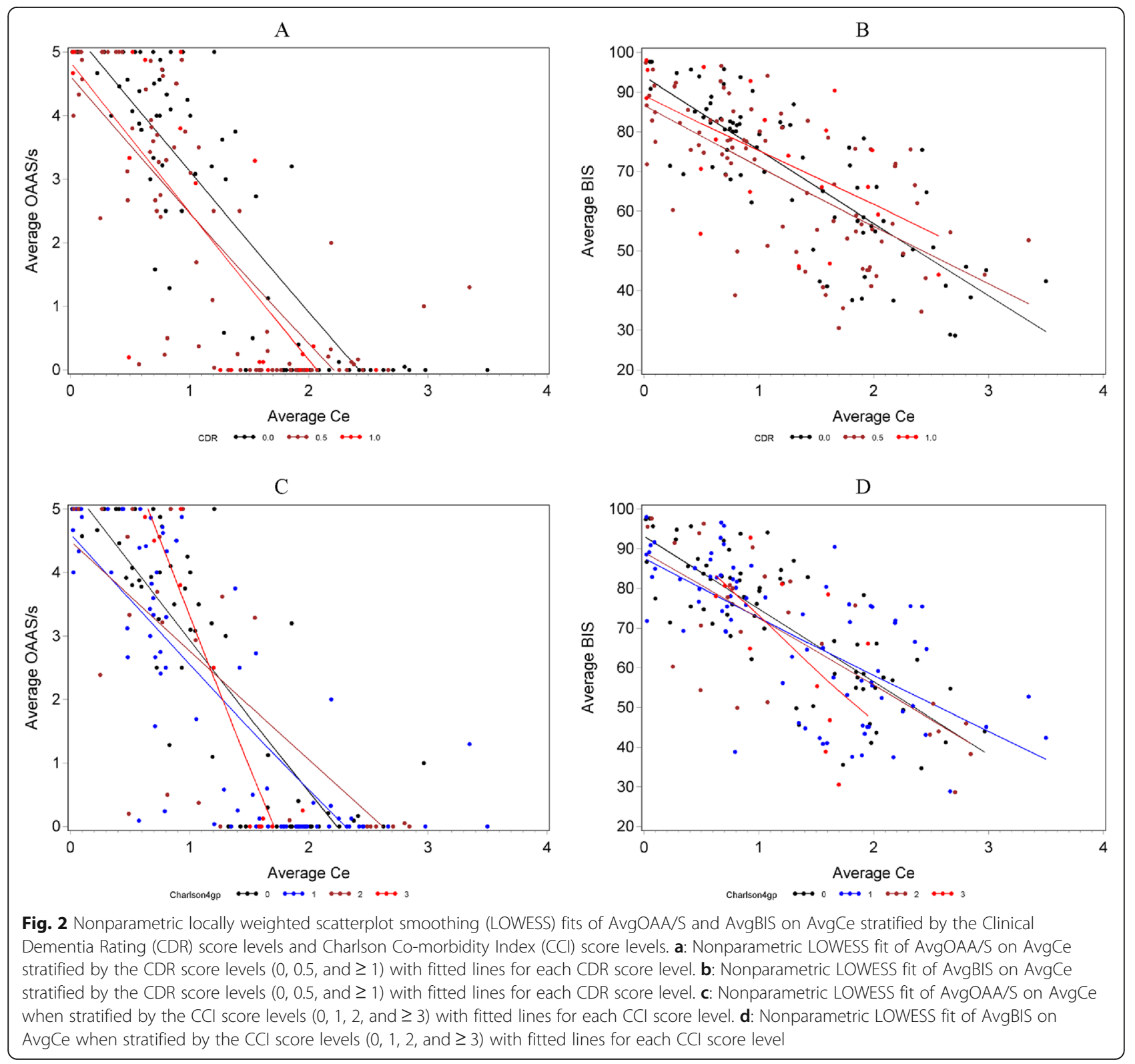


Table 5 Multivariate Regression Analysis for Predictors of AvgOAA/S

\begin{tabular}{lllll}
\hline Parameter & Estimate & Standard Error & t Value & P \\
\hline Intercept & 0.39 & 0.699 & 0.56 & -11.36 \\
AvgCe & -1.55 & 0.137 & 1.69 & 0.57 \\
AvgMAP & 0.01 & 0.007 & 7.15 & 0.001 \\
AvgBIS & 0.04 & 0.006 & 0.83 & $<.0001$ \\
Age & 0.02 & 0.021 & -2.83 & 0.41 \\
AvgCe and Age Interaction & -0.03 & 0.011 & -1.07 & 0.005 \\
Geriatric Depression Score (GDS) & -0.02 & 0.021 & -2.34 & 0.29 \\
GDS and Age Interaction & -0.01 & 0.002 & 0.020 \\
\hline
\end{tabular}

The current study reports that during sedation, the predicted propofol AvgCe affects AvgOAA/S in confirmation of previous reports demonstrating correlations between the blood propofol concentration and OAA/S [27]. AvgOAA/S was affected by an age-AvgCe interaction such that the same level of increase in AvgCe would observe greater decrease in AvgOAA/S score in older patients than in younger patients. This is consistent with Schnider et al. [19] who observed increasing sensitivity to propofol in elderly patients for loss of consciousness. Both age and level of spinal anesthesia have been reported to modify sedation scores [28]. In the current study, age is the more important modifying factor of OAA/S as the overall level of spinal anesthesia demonstrated little variation among participants (T9 \pm 1.5 dermatomes). AvgOAA/S was also predicted by ageGDS interaction such that higher GDS scores in conjunction with age were associated with lower AvgOAA/S score at the same Ce. The GDS is a tool to assess symptoms of depression, and there is evidence to suggest that depression and cognitive impairment may be due to related brain dysfunction [29]. As the interaction between GDS and age in determining OAA/S score have not previously been reported, it will require confirmation. Similar to AvgBIS, AvgOAA/S score was not influenced by any of the baseline cognitive measures performed in the STRIDE study.

\section{Strengths}

Exact amounts and administration time of propofol was recorded allowing for calculation of predicted Ce. Multiple assessments of baseline cognition were performed in this study and all were able to be tested to verify the robustness of our conclusions. Level of sedation was assessed both behaviorally as well as with BIS. Little systematic bias was observed for both means of assessing sedation depth defined by predicted Ce.

\section{Limitations}

Fourteen patients were not included in analysis. However, the baseline characteristics of these 14 patients are comparable to the 186 participants included in this report, and sensitivity analysis demonstrates that the study results would not have been affected by these additional 14 patients on analyses not involving predicted Ce. Only the surgical period was analyzed, and no conclusions can be made concerning hysteresis during induction or emergence which might occur in states of cognitive dysfunction. Reporting the predicted propofol Ce is better than reporting the dose and helps refine our clinical observations. However, without blood concentrations predicted $\mathrm{Ce}$ is subject to bias and inaccuracies which limits the ability to make definitive conclusions concerning pharmacodynamic interactions between cognition and sedation requirements. Although ignoring the variability of individual measurements around the mean level over time during surgery, using the mean levels of Ce, OAA/ $\mathrm{S}$ and BIS during surgery to study their interrelationship with other variables reflects the common practice of maintaining the sedation around a goal level, whether implicitly or not, in clinical management. Lastly, this study performed secondary analysis using existing trial data, with fixed sample size not specifically powered to confirm any hypotheses tested in this study. Although age was identified as significantly modifying the association between $\mathrm{Ce}$ and $\mathrm{OAA} / \mathrm{S}$, we were not able confirm similar effect modification by pre-operative cognitive state measured by CDR score or other baseline cognitive variables using the STRIDE data.

\section{Conclusion}

In summary, during sedation under spinal anesthesia, AvgBIS and AvgOAA/S score are good proxies of predicted AvgCe during surgery in routine clinical practices, as evident in the high correlation and 0 systematic bias demonstrated in the results. Furthermore, the relationships between the predicted AvgCe and both AvgBIS and AvgOAA/S score do not appear to be altered by baseline cognitive impairment. None of the baseline cognitive variables studied in STRIDE independently exerted an effect on either the AvgBIS number or AvgOAA/S score. 


\section{Abbreviations}

AD: Alzheimer's Disease; AvgBIS: Average Bispectral Index; AvgCe: Mean Predicted Ce Level; AvgMAP: Average Mean Arterial Blood Pressure; AvgOAA/S: Average Observer's Assessment of Alertness/Sedation Scale; BA: Bland-Altman; BIS: Bispectral Index; CAM: Confusion Assessment Method; CCl: Charlson Comorbidity Index; CDR: Clinical Dementia Rating; Ce: Predicted Propofol Effect Site Concentration; CSF: Cerebrospinal Fluid; DRS-R-98: Delirium Rating Scale-R-98; DST: Digit Span Test; GDS: Geriatric Depression Scale; LOWESS: Locally Weighted Scatterplot Smoothing; MAP: Mean Arterial Blood Pressure; MMSE: Mini-mental Status Exam; OAA/ S: Observer's Assessment of Alertness/Sedation Scale; SD: Standard Deviations; STRIDE: A Strategy to Reduce the Incidence of Postoperative Delirium in Elderly Patients

\section{Acknowledgments}

The authors would like to thank the assessment team (Kori Kindbom, Rachel Burns, Michael Sklar) for their excellent follow-up of patients, the DSMB members: Jeffery Carson, M.D., Chair; Lee Fleisher, M.D.; Steve Epstein, M.D.; Jay Magaziner, PhD; Anne Lindblad, PhD; and Safety Officer: Sarang Kim, M.D. for their time, effort, and oversight, and Linda Sevier for her expert preparation of the manuscript.

\section{Authors' contributions}

FES - Conception, design, acquisition, analysis, interpretation of data, and drafted and revised the work. KN - Acquisition, interpretation of data, revised the work. ESO- Acquisition, interpretation of data, revised the work. AG Conception, acquisition, analysis, data interpretation, drafted and revised work. NYW - Conception, design, acquisition, analysis, interpretation of data, and drafted and revised the work. All authors approved the submitted version and have agreed to be personally accountable for their contributions and to ensure that questions related to the accuracy or integrity of any part of the work, even ones in which the author was not personally involved, are appropriately investigated, resolved, and the resolution documented in the literature.

\section{Funding}

This research was supported by National Institute of Aging Grant R01 AG033615 and K23AG043504. The funding agency had no role in the design of the study, collection, analysis, and interpretation of data and in writing the manuscript.

\section{Availability of data and materials}

The datasets used and/or analyzed during the current study are available from the corresponding author on reasonable request.

\section{Ethics approval and consent to participate}

Not applicable.

\section{Consent for publication}

Not applicable.

\section{Competing interests}

The authors declare they have no competing interests.

\section{Author details}

'Department of Anesthesiology and Critical Care Medicine, Johns Hopkins Bayview Medical Center, 4940 Eastern Avenue, Baltimore, MD 21224, USA. ${ }^{2}$ Department of Psychiatry and Behavioral Sciences, Johns Hopkins University School of Medicine, A4Center Suite 457, 4940 Eastern Ave, Baltimore, USA. ${ }^{3}$ Division of Geriatric Medicine and Gerontology, Psychiatry and Behavioral Sciences \& Neuropathology, Johns Hopkins University School of Medicine, Mason F. Lord Building, Center Tower, 5200 Eastern Avenue, 7th Floor, Baltimore, MD 21224, USA. ${ }^{4}$ Departments of Anesthesiology and Critical Care Medicine and Neurosurgery, Johns Hopkins Hospital, 1800 Orleans St, Baltimore, MD 21287, USA. ${ }^{5}$ Medicine, Biostatistics and Epidemiology, The Johns Hopkins University, 2024 E. Monument Street, Suite 2-500, Baltimore, MD 21287, USA.
Received: 25 October 2019 Accepted: 20 May 2020

Published online: 28 May 2020

\section{References}

1. Alzheimer's Association. 2018 Alzheimer's disease facts and figures 2018.

2. Alzheimer's disease facts and figures. Alzheimer's \& dementia: the journal of the Alzheimer's Association 2017; 13:325-373.

3. Robinson TN, Wu DS, Pointer LF, Dunn CL, Moss M. Preoperative cognitive dysfunction is related to adverse postoperative outcomes in the elderly. J Am Coll Surg. 2012;215(1):12-7 discussion 17-8.

4. Styra R, Larsen E, Dimas MA, Baston D, Elgie-Watson J, Flockhart L, et al. The effect of preoperative cognitive impairment and type of vascular surgery procedure on postoperative delirium with associated cost implications. J Vasc Surg. 2019;69(1):201-9.

5. Evered LA, Silbert BS, Scott DA, Maruff P, Ames D, Choong PF. Preexisting cognitive impairment and mild cognitive impairment in subjects presenting for total hip joint replacement. Anesthesiology. 2011;114(6):1297-304.

6. Lee HB, Mears SC, Rosenberg PB, Leoutsakos JM, Gottschalk A, Sieber FE. Predisposing factors for postoperative delirium after hip fracture repair in individuals with and without dementia. J Am Geriatr Soc. 2011;59(12):2306-13.

7. Perez-Protto S, Geube M, Ontaneda D, Dalton JE, Kurz A, Sessler DI. Sensitivity to volatile anesthetics in patients with dementia: a case-control analysis. Can J Anaesth. 2014;61(7):611-8.

8. Erdogan MA, Demirbilek S, Erdil F, Aydogan MS, Ozturk E, Togal T, et al. The effects of cognitive impairment on anaesthetic requirement in the elderly. Eur J Anaesthesiol. 2012;29(7):326-31.

9. Glass PS, Bloom M, Kearse L, Rosow C, Sebel P, Manberg P. Bispectral analysis measures sedation and memory effects of propofol, midazolam, isoflurane, and alfentanil in healthy volunteers. Anesthesiology. 1997;86(4): 836-47.

10. Sieber FE, Neufeld KJ, Gottschalk A, Bigelow GE, Oh ES, Rosenberg PB, et al. Effect of depth of sedation in older patients undergoing hip fracture repair on postoperative delirium: the STRIDE randomized clinical trial. JAMA Surg. 2018,8.

11. Sieber F, Neufeld KJ, Gottschalk A, Bigelow GE, Oh ES, Rosenberg PB, et al. Depth of sedation as an interventional target to reduce postoperative delirium: mortality and functional outcomes of the strategy to reduce the incidence of postoperative delirium in elderly patients randomized clinical trial. Br J Anaesth. 2019;122(4):480-9.

12. Li T, Wieland LS, Oh E, Neufeld KJ, Wang NY, Dickersin K, et al. Design considerations of a randomized controlled trial of sedation level during hip fracture repair surgery: a strategy to reduce the incidence of postoperative delirium in elderly patients. Clin Trials 2017 Jan 01:1740774516687253supplemental material.

13. Folstein MF, Folstein SE, McHugh PR. "Mini-mental state". A practical method for grading the cognitive state of patients for the clinician. J Psychiatr Res. 1975;12(3):189-98.

14. Oh ES, Blennow K, Bigelow GE, Inouye SK, Marcantonio ER, Neufeld KJ, et al. Abnormal CSF amyloid-beta42 and tau levels in hip fracture patients without dementia. PLoS One. 2018;13(9):e0204695.

15. Yesavage JA, Sheikh J. 9/Geriatric Depression Scale (GDS). Clin Gerontol 1986 11/18; 5(1-2):165-173.

16. Charlson ME, Pompei $P$, Ales $K L$, Mackenzie CR. A new method of classifying prognostic comorbidity in longitudinal studies: development and validation. J Chronic Dis. 1987:40(5):373-83.

17. Inouye SK, van Dyck CH, Alessi CA, Balkin S, Siegal AP. RI. H. Clarifying confusion: the confusion assessment method. A new method for detection of delirium. Ann Intern Med. 1990;113(12):941-8.

18. Trzepacz PT, Mittal D, Torres R, Kanary K, Norton J, Jimerson N. Validation of the delirium rating scale-revised-98: comparison with the delirium rating scale and the cognitive test for delirium. J Neuropsychiatry Clin Neurosci. 2001;13(2):229-42.

19. Schnider TW, Minto CF, Shafer SL, Gambus PL, Andresen C, Goodale DB, et al. The influence of age on propofol pharmacodynamics. Anesthesiology. 1999;90(6):1502-16.

20. Rasmussen LS, Christiansen M, Rasmussen H, Kristensen PA, JT M. Do blood concentrations of neurone specific enolase and S-100 beta protein reflect cognitive dysfunction after abdominal surgery? ISPOCD Group Br J Anaesth. 2000;84(2):242-4.

21. Liu SH, Wei W, Ding GN, Ke JD, Hong FX, Tian M. Relationship between depth of anesthesia and effect-site concentration of propofol during 
induction with the target-controlled infusion technique in elderly patients Chin Med J. 2009;122(8):935-40.

22. Kazama T, Ikeda K, Morita K, Kikura M, Doi M, Ikeda T, et al. Comparison of the effect-site $k(\mathrm{eO}) \mathrm{s}$ of propofol for blood pressure and EEG Bispectral index in elderly and younger patients. Anesthesiology. 1999;90(6):1517-27.

23. Myles PS. Bispectral index monitoring in ischemic-hypoxic brain injury. J Extra Corpor Technol 2009; 41(1):P15-9.

24. Merat S, Levecque JP, Le Gulluche Y, Diraison Y, Brinquin L, Hoffmann JJ. BIS monitoring may allow the detection of severe cerebral ischemia. Can J Anaesth. 2001;48(11):1066-9.

25. Win NN, Kohase H, Miyamoto T, Umino M. Decreased bispectral index as an indicator of syncope before hypotension and bradycardia in two patients with needle phobia. Br J Anaesth. 2003;91(5):749-52.

26. Lee SW, Choi SE, Han JH, Park SW, Kang WJ, Choi YK. Effect of beach chair position on bispectral index values during arthroscopic shoulder surgery. Korean J Anesthesiol. 2014;67(4):235-9.

27. Casati A, Fanelli G, Casaletti E, Colnaghi E, Cedrati V, Torri G. Clinical assessment of target-controlled infusion of propofol during monitored anesthesia care. Can J Anaesth. 1999;46(3):235-9.

28. Roh GU, Kim Y, Ha SH, Jeong KH, Choi S, Han DW. Modelling of the sedative effects of Propofol in patients undergoing spinal Anaesthesia: a Pharmacodynamic analysis. Basic Clin Pharmacol Toxicol. 2016;1 18(6):480-6.

29. Morimoto SS, Alexopoulos GS. Cognitive deficits in geriatric depression: clinical correlates and implications for current and future treatment. Psychiatr Clin North Am. 2013;36(4):517-31.

\section{Publisher's Note}

Springer Nature remains neutral with regard to jurisdictional claims in published maps and institutional affiliations.

Ready to submit your research? Choose BMC and benefit from:

- fast, convenient online submission

- thorough peer review by experienced researchers in your field

- rapid publication on acceptance

- support for research data, including large and complex data types

- gold Open Access which fosters wider collaboration and increased citations

- maximum visibility for your research: over $100 \mathrm{M}$ website views per year

At $\mathrm{BMC}$, research is always in progress.

Learn more biomedcentral.com/submissions 